\title{
Longitudinal Analysis of Sleep Spindle Maturation from Childhood through Late Adolescence
}

\author{
Zoey Y. Zhang, ${ }^{1}$ Ian G. Campbell, ${ }^{1}$ Pari Dhayagude, ${ }^{1}$ Harrison C. Espino, ${ }^{2}$ and Irwin Feinberg ${ }^{1}$ \\ ${ }^{1}$ Department of Psychiatry and Behavioral Sciences, University of California Davis, Sacramento, California 95817, and ${ }^{2}$ Department of Computer \\ Science, University of California Davis, Davis, California 95616
}

Sleep spindles are intermittent bursts of 11-15 Hz EEG waves that occur during non-rapid eye movement sleep. Spindles are believed to help maintain sleep and to play a role in sleep-dependent memory consolidation. Here we applied an automated sleep spindle detection program to our large longitudinal sleep EEG dataset (98 human subjects, 6-18 years old, $>2000$ uninterrupted nights) to evaluate maturational trends in spindle wave frequency, density, amplitude, and duration. This large dataset enabled us to apply nonlinear as well as linear age models, thereby extending the findings of prior cross-sectional studies that used linear models. We found that spindle wave frequency increased with remarkable linearity across the age range. Central spindle density increased nonlinearly to a peak at age 15.1 years. Central spindle wave amplitude declined in a sigmoidal pattern with the age of fastest decline at 13.5 years. Spindle duration decreased linearly with age. Of the four measures, only spindle amplitude showed a sex difference in dynamics such that the age of most rapid decline in females preceded that in males by 1.4 years. This amplitude pattern, including the sex difference in timing, paralleled the maturational pattern for $\delta(1-4 \mathrm{~Hz})$ wave power. We interpret these age-related changes in spindle characteristics as indicators of maturation of thalamocortical circuits and changes in sleep depth. These robust age-effects could facilitate the search for cognitive-behavioral correlates of spindle waveforms and might also help guide basic research on EEG mechanisms and postnatal brain maturation.

Key words: adolescence; EEG; longitudinal; maturation; sleep spindle

Significance Statement

The brain reorganization of adolescence produces massive changes in sleep EEG. These changes include the morphology and abundance of sleep spindles, an EEG marker of non-rapid eye movement sleep believed to reflect offline memory processes and/or protection of the sleep state. We analyzed $>2000$ nights of longitudinal sleep EEG from 98 subjects (age 6-18 years old) to investigate maturational changes in spindle amplitude, frequency, density, and duration. The large dataset enabled us to detect nonlinear as well as linear age changes. All measures showed robust age effects that we hypothesize reflect the maturation of thalamocortical circuits and decreasing sleep depth. These findings could guide further research into the cognitivebehavioral correlates of sleep spindles and their underlying brain mechanisms.

\section{Introduction}

The human brain undergoes a pervasive reorganization during adolescence (Feinberg, 1982/1983). This reorganization appears to be the final iteration of a strategy nature uses repeatedly in the construction of vertebrate nervous systems: an initial overproduction followed by reduction and refine-

Received Sep. 17, 2020; revised Mar. 9, 2021; accepted Mar. 18, 2021.

Author contributions: Z.Y.Z., I.G.C., P.D., and H.C.E. analyzed data; Z.Y.Z. wrote the first draft of the paper; Z.Y.Z., I.G.C., P.D., H.C.E., and I.F. edited the paper; I.G.C. and I.F. designed research; I.G.C. and I.F. performed research.

This work was supported by U.S. Public Health Service Grants R01HL116490 and R01MH062521.

The authors declare no competing financial interests.

Correspondence should be addressed to lan G. Campbell at igcampbell@ucdavis.edu.

https://doi.org/10.1523/JNEUROSCI.2370-20.2021

Copyright $\odot 2021$ the authors ment by regressive processes such as synaptic elimination (Purves, 1988).

Changes in the EEG patterns of sleep are among the most noteworthy adolescent brain changes. They are useful because they directly reflect neuronal activity and can be measured objectively and noninvasively (and hence longitudinally). Moreover, they are, along with cortical metabolic rate (Chugani et al., 1987), among the largest changes in adolescent physiology known to occur in the human brain. Some of these electrophysiological changes have been found to be correlated with structural changes apparent in MRI (Buchmann et al., 2010; Goldstone et al., 2018). Among the maturational changes in sleep EEG waveforms, those in sleep spindles are of special interest. Sleep spindles are bursts of sinusoidal $12-15 \mathrm{~Hz}$ waves $(11-15 \mathrm{~Hz}$ in children) that occur several times per minute in non-rapid eye movement (NREM) sleep (see example in Fig. 1). They are 
widely distributed across the scalp and show anterior-posterior gradients. Practically, they are crucial in distinguishing low amplitude non-REM EEG from otherwise similar REM EEG in both visual and automated sleep stage scoring. Sleep spindles have been proposed to play a sleep protective role by inhibiting sensory information (De Gennaro and Ferrara, 2003; Luthi, 2014), and they are a focus of research on memory processing during sleep (Fogel and Smith, 2011).

Uchida et al. (1991) recognized that, because the amplitudes of waves in organized spindle bursts are higher than those of background $12-15 \mathrm{~Hz}$ EEG, fast Fourier transform (FFT) power in $12-15 \mathrm{~Hz}(\sigma)$ is dominated by the contribution of organized spindles. We have previously used $\sigma$ power to index overall spindle activity and to estimate its change across adolescence (Campbell and Feinberg, 2016). However, FFT power does not distinguish elements of sleep spindle activity that could be biologically important. Pattern-recognition methods are required to determine maturational changes in such aspects of spindle "microstructure" as average frequency, wave amplitude, density of bursts, and their durations. Prior cross-sectional studies have found that sleep spindle amplitude and duration decrease across adolescence, whereas spindle wave frequency and density increase (Purcell et al., 2017; Goldstone et al., 2019).

In this paper, we report the first extensive longitudinal measurements of these aspects of spindle microstructure across human adolescence. We applied a modification of the pattern recognition methods of Goldstone et al. (2019) to evaluate maturational changes in the spindle features. Our longitudinal dataset consists of $\sim 2000$ nights of EEG recordings from 98 participants, ranging from 6 to 18 years of age. This large dataset allowed us to evaluate linear and nonlinear models of the maturational changes in the following sleep spindle characteristics: average frequency of waves within the sleep spindle, peaktrough wave amplitude, sleep spindle density, and sleep spindle duration. We also examined sex-related differences in spindle maturation and differences in frontal and central spindle maturation.

\section{Materials and Methods}

Subjects and experimental design. We applied sleep spindle detection and analysis methods to previously published longitudinal data from 98 subjects (47 female, 51 male) spanning ages 5.96-18.40 years (Feinberg and Campbell, 2013). The study followed participants in three cohorts: C6, C9, and C12. Subjects in the C6 cohort (11 female, 17 male) were recruited at $\sim 6$ years old and stayed in the study for up to 4 years. Subjects in the C 9 cohort ( 17 female, 15 male) were recruited at $\sim 9$ years old and stayed in the study for up to 7 years. Subjects in the C12 cohort (19 female, 19 male) were recruited at $\sim 12$ years of age and stayed in the study for up to 6 years. Thus, the C6 cohort had 1 year of age overlap (910 years old) with the $\mathrm{C} 9$ cohort, and the $\mathrm{C} 9$ cohort had 4 years of age overlap (12-16 years old) with the $\mathrm{C} 12$ cohort.

Participants in the C6 cohort completed two consecutive all-night EEG recordings every 6 months with their usual school year weeknight sleep schedules. Subjects in the C9 and C12 cohorts completed four consecutive all-night EEG recordings every 6 months. For the first and second nights, participants in the $\mathrm{C} 9$ and $\mathrm{C} 12$ cohorts were instructed to maintain their habitual school year weeknight sleep schedules. On the third and fourth nights, participants in the C9 and C12 cohorts retired at their habitual weekday bedtime but were asked to sleep as long as possible. This paper focuses on maturation of spindles on a night of sleep on the habitual sleep schedule. For the current analyses, night 4 data were excluded because sleep extension on night 3 would have affected the sleep EEG on night 4 . Night 3 data are included because the first $5 \mathrm{~h}$ of NREM sleep (see below) occur before the period of sleep extension. Further enrollment and experiment details have been previously published (Feinberg and Campbell, 2013).

EEG recording and analysis. EEG was recorded in subjects' homes in their habitual sleep environments, with 1-cm-diameter gold cup electrodes filled with Ten 20 conductive paste applied at the following locations: $\mathrm{C} 3, \mathrm{C} 4, \mathrm{Fz}, \mathrm{Cz}, \mathrm{O} 1, \mathrm{O} 2$ (or $\mathrm{Pz}$ in later recordings), $\mathrm{A} 1, \mathrm{~A} 2, \mathrm{LOC}$, and ROC. All signals were recorded versus reference. Bipolar montages (C3/ $\mathrm{A} 2, \mathrm{C} 4 / \mathrm{A} 1, \mathrm{Fz} / \mathrm{A} 2, \mathrm{Fz} / \mathrm{A} 1)$ were obtained by subtraction. The first nine semiannual recordings in the $\mathrm{C} 9$ and $\mathrm{C} 12$ cohorts were recorded on Grass $\mathrm{H} 2 \mathrm{O}$ ambulatory recorders $(200 \mathrm{~Hz}$ digitization rate). After Grass discontinued support for the $\mathrm{H} 2 \mathrm{O}$, the rest of the recordings in $\mathrm{C} 9$ and C12 and all recordings in the C6 cohort were recorded on Grass Aura ambulatory recorders $(400 \mathrm{~Hz}$ digitization rate). The $\mathrm{H} 2 \mathrm{O}$ and Aura recorders both use the same single-pole low-frequency filter with a $-3 \mathrm{~dB}$ point at $0.5 \mathrm{~Hz}$ and a $6 \mathrm{~dB} /$ octave slope. The high-frequency filter for the Aura is a 3 pole filter with a $-3 \mathrm{~dB}$ point at $100 \mathrm{~Hz}$ and an $18 \mathrm{~dB} /$ octave slope. The documentation for the $\mathrm{H} 2 \mathrm{O}$ does not list the specifics for the high-frequency filter. Our frequency response curve found the $-3 \mathrm{~dB}$ point to be at $55 \mathrm{~Hz}$. The frequency response curves for both recorders are included as a supplemental figure to a previous publication (Campbell et al., 2011). The hardware filter on the $\mathrm{H} 2 \mathrm{O}$ slightly reduced the amplitude of waves within the frequency range of sleep spindles, from a $0.66 \%$ reduction at $9 \mathrm{~Hz}$ to a $1.89 \%$ reduction at $15 \mathrm{~Hz}$. Frequency response curves were established for both the $\mathrm{H} 2 \mathrm{O}$ and Aura amplifiers by recording $200 \mu \mathrm{V}$ sine waves of various frequencies. Comparing the curves produced the following quadratic equation for a correction factor for $\mathrm{H} 2 \mathrm{O}$ recording amplitude: correction factor $=$ 
$0.000094(\text { freq })^{2}-0.00021$ (freq) +1.0009 . Average peak trough amplitude (see below) for each night of EEG recorded on an $\mathrm{H} 2 \mathrm{O}$ was adjusted by multiplying by the correction factor based on the mean spindle wave frequency for that recording.

Trained laboratory personnel scored all EEG recordings for sleep stages using a visual display (PASSPLUS, Delta Software) of the digitized central and occipital EEG and EOG data. The scorers first chose a primary analysis channel, either C3/A2 or C4/A1, by visually selecting the channel with fewer artifacts. Then the scorers rated each $20 \mathrm{~s}$ epoch as wake, NREM, REM or movement based on Rechtschaffen and Kales (1968) criteria. The presence of artifacts on the primary analysis channel was also noted. All EEG recordings were later processed by an anonymization program, which converted EEG data in Grass TWin format to EDF format and converted PASSPLUS scoring files from DBF format to CSV format.

Spindle detection and analysis. We used an automated spindle detection MATLAB (The MathWorks) program adapted from the program used by Goldstone et al. (2019). This program was built on several previously published spindle detection methods (Bodizs et al., 2009; Andrillon et al., 2011). For each subject-night, spindles were analyzed on a single central (C3-A2 or C4-A1) and a single frontal (Fz-A2 or Fz-A1) with the choice between signals (e.g., C3-A2 or C4-A1) based on which had fewer artifacts. The individual spindle frequency range for each subject's recording was defined by using selected central and frontal channels. EEGLAB toolbox (Delorme and Makeig, 2004) produced power spectra $(0.3-80 \mathrm{~Hz})$ for each artifact-free NREM epoch using fast Fourier Transform of central and frontal EEG data in $5.12 \mathrm{~s}$ Hanning windows (producing $0.195 \mathrm{~Hz}$ bins). The $\sigma$ peaks $(9-16 \mathrm{~Hz}$ ) of the frontal (slow spindle frequency) and central channels (fast spindle frequency) FFT power spectra were detected with MATLAB 2019a findpeaks function with MinPeakDistance $=0.39 \mathrm{~Hz}$ and MinPeakHeight $=1$ natural $\log$ unit. For each subject, the frontal spindle frequency range was defined as $\pm 1.5 \mathrm{~Hz}$ of the slow-frequency peak, and the central spindle frequency range was defined as $\pm 1.5 \mathrm{~Hz}$ of the fast-frequency peak (Goldstone et al., 2019). The spindle frequency range was used to bandpass filter the raw EEG. The filtered EEG was then processed by Hilbert transformation to obtain the instantaneous amplitude envelope (Goldstone et al., 2019). The spindle detection threshold was defined as 3 SDs above the mean Hilbert envelope, and the spindle rejection threshold was defined as 10 SDs above the mean (Andrillon et al., 2011; Goldstone et al., 2019).

The MATLAB program we adapted from Goldstone et al. (2019) produced the following measurements: spindle duration, spindle Hilbert amplitude, spindle density, fast spindle frequency, and slow spindle frequency. The spindle duration and Hilbert amplitude were calculated as the average of each spindle's duration and amplitude across the NREM artifact-free epochs. The spindle density was calculated as the average number of spindles per minute of NREM artifact-free sleep. As mentioned before, the fast and slow spindle frequencies were identified for central and frontal channels, respectively, as the frequency at which the power spectra peaked within the $\sigma$ frequency range.

Although sleep spindles are the dominant EEG pattern within the $\sigma$ band, the spectral peak is not a direct measure of spindle frequency, nor is Hilbert amplitude an easily grasped, intuitive amplitude measure. Thus, in addition to the five measurements from the Goldstone et al. (2019) method, two more measurements were added to the final output: spindle peak-trough amplitude and spindle frequency.

The program used MATLAB's 2019a findpeaks function on the bandpass filtered EEG, with MinPeakDistance $=50 \mathrm{~ms}$ and MinPeakProminence $=$ $5 \mu \mathrm{V}$, to identify the amplitudes of peaks and troughs. We then arranged these amplitudes sequentially and calculated the peak-trough amplitude as the averaged amplitude difference between each sorted amplitude of peak and trough. For example, in Figure 1 (bottom), the amplitude difference between $\operatorname{Tr} 0$ and $\mathrm{P} 0$ is $40.62 \mu \mathrm{V}$. This value was averaged with the amplitude difference between $\mathrm{P} 0$ and $\mathrm{Tr} 1, \mathrm{~T} 1$ and $\mathrm{P} 1, \mathrm{P} 1$ and Tr2, etc. through the final difference between Tr14 and P14 to find the average peak trough amplitude of this spindle. The spindle peak-trough amplitude across artifact-free NREM was then calculated as the average of the average amplitude of each spindle.

The program calculated frequency by first counting (starting with 0 ) the number of peaks within a spindle. Then the total peak count was divided by the time between the first and last peak. Again, using Figure 1 (bottom) as an example, P0 occurs at $13.95 \mathrm{~s}$ and P14 occurs at $15.03 \mathrm{~s}$; $14 / 1.08=12.96 \mathrm{~Hz}$. This frequency was averaged across all spindles in artifact-free NREM sleep.

Spindle measures change across the night, and sleep duration in a night changes with age. To overcome this age-related change in sleep duration, we analyzed the first $5 \mathrm{~h}$ of NREM sleep, as we have in previous publications from this study.

Artifact rejection. The first step in artifact rejection was to eliminate nights with hand scored artifacts in $>10 \%$ of epochs. Because the score files only had artifact information for central electrodes, we conducted FFT on Fz referred to A1 and A2 and plotted the results to decide which derivations were more contaminated with noise (i.e., a distinct high peak at 20, 40, 60, or $80 \mathrm{~Hz}$ ). For most recordings, at least one frontal derivation was free of noise. If neither derivation was noise free, the night was excluded from further analysis. We also noticed that many recordings with outlier spindle measures had an elevated ratio of SD to mean of the night's Hilbert envelopes. Thus, recordings with ratio $>0.95$ were also excluded. Elimination of poor recordings left 2030 nights of central data and 1911 nights of frontal data for analysis. For all usable recordings, the epochs that were hand marked as artifact-free were selected for both frontal and central channels for the analysis.

We later noticed that the Aura recordings with a $400 \mathrm{~Hz}$ digitization rate had spindle durations $\sim 8 \%$ lower than recordings digitized at $200 \mathrm{~Hz}$. To eliminate this discrepancy and other sample rate-related spindle measure differences, we reduced the $400 \mathrm{~Hz}$ recordings to $200 \mathrm{~Hz}$ by removing every other sample from the digitized dataset in MATLAB.

Statistical analysis. Before statistical analysis, all spindle characteristics for nights 1, 2, and 3 at each semiannual recording were averaged for each subject for central and frontal channels. Age-related trends in spindle measures were determined separately for central and frontal channels with SAS mixed effects analysis (Singer, 1998).

For each measure, we first analyzed the linear trend and then determined whether the fit (evaluated with Akaike information criterion [AIC] and Bayesian information criterion [BIC]) improved with addition of quadratic (age squared) and then cubic terms (age cubed). If significant curvature was detected with quadratic and/or cubic terms, we tested whether saturating exponential or logistic curves improved the fit. We tested the fit of both a simple logistic curve $\left[\mathrm{Y}=\mathrm{D}-\mathrm{A}^{*}\left(1 /\left(1+\mathrm{e}^{-\mathrm{c}_{*}(\text { age }-\mathrm{M})}\right)\right)\right]$ and a Gompertz curve $\left[\mathrm{Y}=\mathrm{D}-\mathrm{A}^{*} \mathrm{e}^{-\mathrm{e}^{-e^{*}(a g e-\mathrm{M})}}\right]$. We previously found that a Gompertz curve best fit the adolescent decline in $\delta$ (1-4 Hz) EEG power (Campbell and Feinberg, 2009). Once the form with the best fit was identified, sex was added to the model to determine whether the age-related changes differed between boys and girls.

Because of the fewer number of nights recorded for the C6 cohort, there were more missing data points where neither night was usable because of recording problems or artifacts. Therefore, we are more confident in the maturational trends across the 9 to 18 year age range of the C9 and C12 cohorts. Furthermore, in previously published analyses (Feinberg and Campbell, 2013), we observed different maturational trends at the younger end of the age range. Therefore, we initially analyzed data for the $\mathrm{C} 9$ and $\mathrm{C} 12$ cohorts, and then reexamined the trends with all cohorts $(\mathrm{C} 6, \mathrm{C} 9, \mathrm{C} 12)$ together.

For spindle duration, the age trend was noticeably different for nights recorded with Grass $\mathrm{H} 2 \mathrm{O}$ recorders and nights recorded on Grass Aura recorders. Thus, we analyzed the age trend of spindle durations with all recordings and with Aura recordings excluded.

\section{Results}

\section{Central spindle measures: changes from 9 to 18 years of age}

An initial linear analysis of age effects on central channel spindle characteristics showed significant age effects on spindle frequency $\left(F_{(1,716)}=337, p<0.0001\right)$, spindle density $\left(F_{(1,716)}=\right.$ 7.28, $p=0.0071)$, spindle duration $\left(F_{(1,716)}=46.1, p<0.0001\right)$, and spindle peak-trough amplitude $\left(F_{(1,716)}=149, p<0.0001\right)$.

For spindle frequency, the best fit model for age effect was a linear model. BIC was lowest for the linear model (Table 1). For 
Table 1. Fit measures for models describing the maturation of sleep spindle characteristics

\begin{tabular}{|c|c|c|c|c|c|c|c|}
\hline Spindle measure & Fit measure & Linear & Quadratic & Cubic & Saturating exponential & Logistic & Gompertz \\
\hline \multirow[t]{2}{*}{ Frequency } & $\mathrm{AlC}$ & -82.9 & -81.2 & $-85.0^{a}$ & -10.2 & - & - \\
\hline & $\mathrm{BIC}$ & $-69.4^{a}$ & -65.5 & -67.0 & 1.1 & - & - \\
\hline \multirow[t]{2}{*}{ Density } & AIC & 696.6 & $681.3^{a}$ & 683.2 & 698.5 & - & - \\
\hline & $\mathrm{BIC}$ & 710.1 & $697.1^{a}$ & 701.2 & 714.3 & - & - \\
\hline \multirow[t]{2}{*}{ Amplitude } & AIC & 4398.4 & 4396.5 & 4348.2 & - & 4330.5 & $4323.3^{a}$ \\
\hline & BIC & 4411.9 & 4412.2 & 4366.1 & - & 4355.5 & $4348.0^{a}$ \\
\hline
\end{tabular}

Models were fit to data from the $\mathrm{C} 9$ and $\mathrm{C} 12$ cohorts. Fit measures are Akaike information criterion - AIC and Bayesian information criterion - BIC. A lower number is a better fit. Best fit is indicated with superscript "a".

AIC, there was a small improvement in fit with a cubic model, but the improvement in AIC was not as great as the worsening in BIC. Spindle frequency (Fig. 2A) increased significantly $\left(F_{(1,716)}=337\right.$, $p<0.0001$ ) by $0.119 \mathrm{~Hz}$ per year from an intercept of $11.47 \mathrm{~Hz}$ at age 6 .

Rather than increasing linearly, spindle density increased to a peak before declining. Both AIC and BIC show that a quadratic model best fit the age effects on spindle density (Table 1). Central spindle density peaked at 4.90 spindles $/ \mathrm{min}\left(t_{(68)}=\right.$ $109, p<0.0001)$ at age 15.15 years $\left(t_{(68)}=\right.$ $30.1, p<0.0001$ ) (Fig. 2B).

Spindle peak-trough amplitude values were fairly stable between ages 9 and 12 years before decreasing steeply between ages 12 and 16 years, after which the rate of decline slowed (Fig. 2C). This type of age trend is modeled with logistic curves. Specifically, BIC and AIC values for the Gompertz model showed the best fit for the age-related decline in spindle amplitude. The upper asymptote was $40.65 \mu \mathrm{V}$ $\left(t_{(67)}=40.6, p<0.0001\right)$ and amplitude decreased $10.31 \mu \mathrm{V}\left(t_{(67)}=11.1, p<\right.$ $0.0001)$ to the lower asymptote. The age of most rapid decline was 13.49 years $\left(t_{(67)}=\right.$ 62.8, $p<0.0001$ ).

As shown in Figure $2 D$, the age trajectory for spindle duration changed at age 14 for the C9 cohort and at age 16 for the C12 cohort. These ages corresponded to the change from the $\mathrm{H} 2 \mathrm{O}$ to Aura recorders. We analyzed a simple linear trend with and without the data from the Aura recorders. With all recordings, central EEG spindle duration for the C9 and C12 cohorts decreased slightly, $6.53 \mathrm{~ms} / \mathrm{yr}$ but significantly $\left(F_{(1,716)}=46.1, p<0.0001\right)$ from an intercept of $920.3 \mathrm{~ms}$ at age 6. Excluding Aura recordings slightly increased the intercept $(931.6 \mathrm{~ms})$ and slope $(-8.31 \mathrm{~ms} / \mathrm{yr})$.

Central spindle measures: changes from 6 to 18 years of age

Including data from the $\mathrm{C} 6$ cohort did not markedly alter the overall maturational
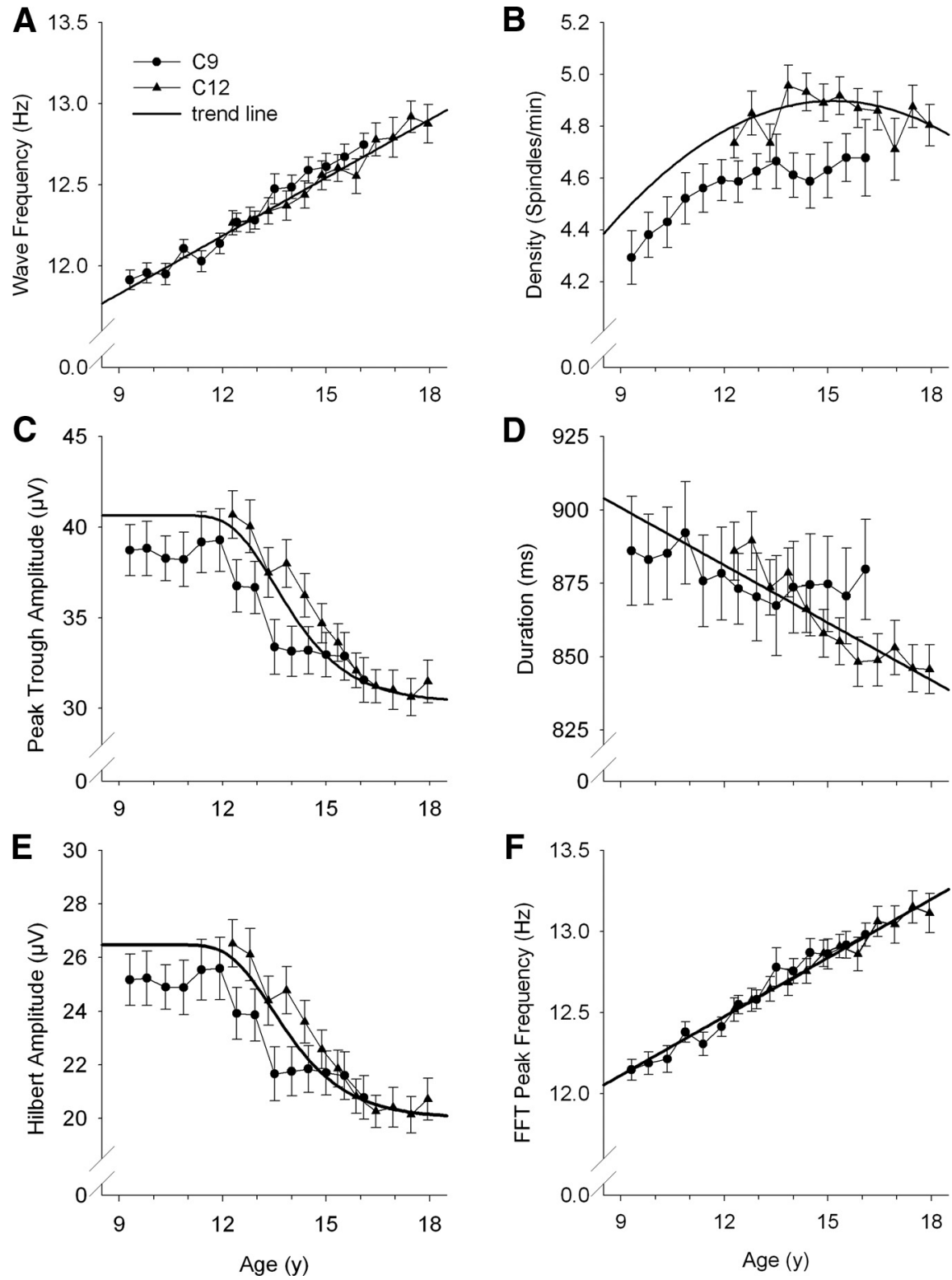

Figure 2. The maturation of six central spindle measures across ages 9-18 years. Each dot represents the mean \pm SE for the $C 9$ (circles) and $\mathrm{C} 12$ (triangles) age cohorts at each semiannual recording. For each measure, a trend line is plotted for the equation that best fits the change with age. Spindle wave frequency $(\boldsymbol{A})$ increased linearly with age. Spindle density $(\boldsymbol{B})$ maturation was described by a parabola that peaked at $\sim 15$ years of age. Spindle peak-trough amplitude $(\boldsymbol{C})$ declined from an upper asymptote with an age of most rapid decline at 13.49 years. Spindle duration $(\boldsymbol{D})$ decreased linearly with age. The change in recorders during the 10th recording may have affected the maturational trend in duration. The maturational trend for Hilbert amplitude $(\boldsymbol{E})$ closely resembled that for peak-trough amplitude, and the trend for fast spindle frequency $(\boldsymbol{F})$ closely resembled that for spindle wave frequency. 

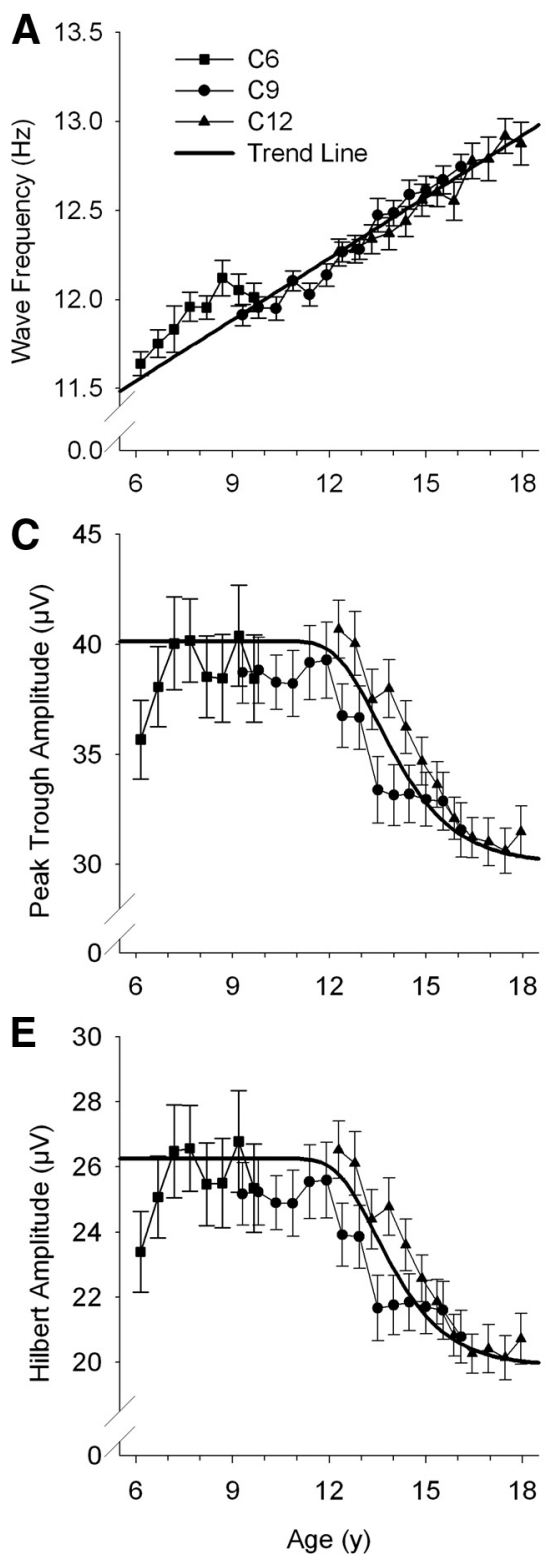
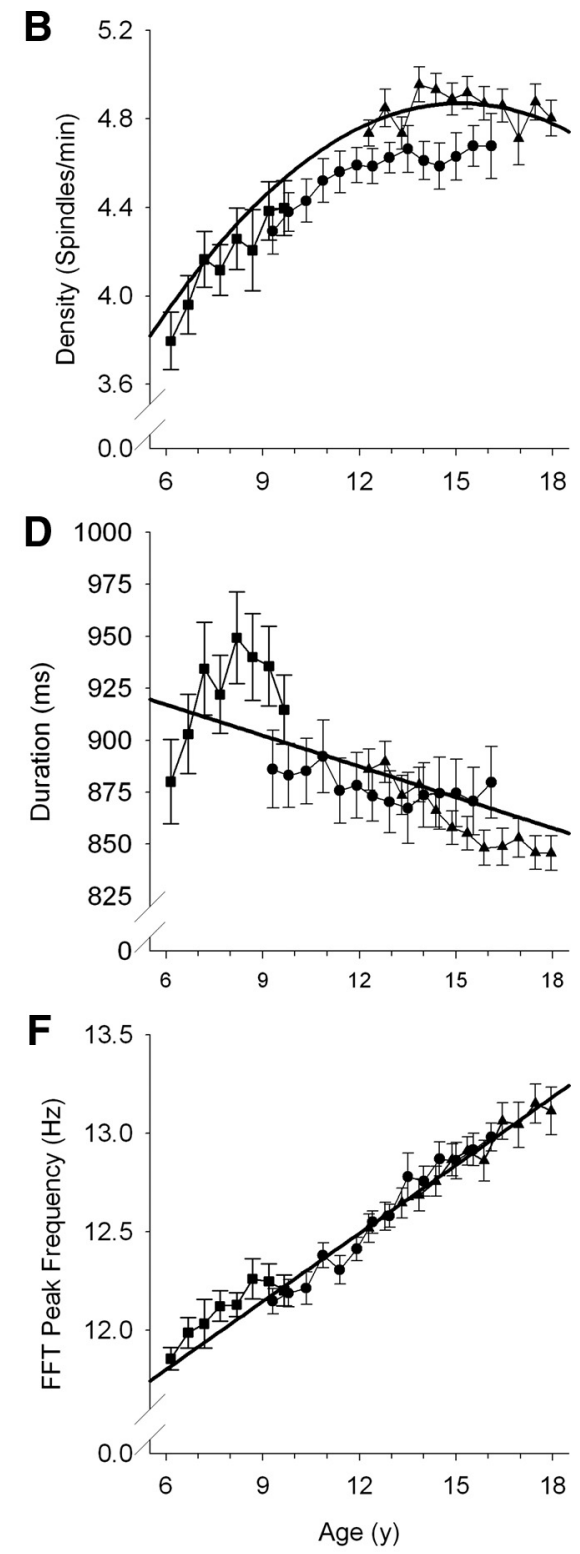

Figure 3. The maturation of six central spindle measures across ages $6-18$ years. Format is the same as in Figure 2 , but this figure adds data for the (6 cohort (squares). Trend lines for spindle wave frequency $(\boldsymbol{A})$, spindle density $(\boldsymbol{B})$, peak trough amplitude $(\boldsymbol{C})$, spindle duration $(\boldsymbol{D})$, Hilbert amplitude $(\boldsymbol{E})$, and sigma fast peak frequency $(\boldsymbol{F})$ fit to data for all three age cohorts closely resemble the trend lines for the $\mathrm{C} 9$ and $\mathrm{C} 12$ data in Figure 2.

trends of any of the central spindle measures (Fig. 3). For each measure, the parameter estimates for equations that describe the age-related changes were within the $95 \%$ CIs for the parameter estimates produced by analysis of data from the C9 and C12 cohorts alone (Table 2). However, there were some points where the data from the younger cohort deviated from the maturational trends. For example, spindle frequency at each recording for the $\mathrm{C} 6$ cohort was above the linear trend line that describes the age-related increase (Fig. $3 A$ ). Peak trough amplitude increased over the first three recordings before declining slightly at the fifth recording at the age of $\sim 8$ years (Fig. $3 B$ ). A post hoc analysis of all recordings with participant age $<8$ years showed a significant $(p=0.0045)$ age-related increase in amplitude. The simple linear trend for spindle duration did not adequately describe the age trend for the $\mathrm{C} 6$ cohort, which increased to a peak at age 8 before declining.

\section{Difference between frontal and central spindles}

For the frontal channel (Fz/A1 or Fz/A2), an initial linear analysis showed significant age effects on spindle frequency $\left(F_{(1,682)}=41.4, p<0.0001\right)$, spindle density $\left(F_{(1,682)}=4.82, p=0.029\right)$, spindle duration $\left(F_{(1,682)}=39.2, p<0.0001\right)$, and spindle peak-trough amplitude $\left(F_{(1,682)}=\right.$ 237, $p<0.0001)$. The age models determined from the central EEG (i.e., linear, quadratic, Gompertz) were fit to the frontal EEG data.

Table 3 and Figure 4 compare the maturational trends for frontal spindle measures to those for central spindle measures for the $\mathrm{C} 9$ and $\mathrm{C} 12$ cohorts. Frontal spindles had a lower frequency than did central spindles. The age 6 intercept for frontal spindle frequency was $10.87 \mathrm{~Hz}$ versus $11.47 \mathrm{~Hz}$ for central spindles. The age-related frequency increase in frontal spindles was similar to that for central $(0.099$ vs $0.119 \mathrm{~Hz} / \mathrm{yr})$. The density of frontal spindles was very similar to that of central spindles. The age of peak density was earlier for frontal than central (14.53 vs 15.15 ), but the $95 \%$ CIs (Table 3 ) overlapped. Amplitude was greater for frontal spindles (upper asymptote $=59.17$ vs $40.65 \mu \mathrm{V}$ ) and the age-related decrease was greater (decrease to lower asymptote= 22.88 vs $10.31 \mu \mathrm{V})$, but the age of most rapid decline was similar for frontal and central (13.58 vs 13.49 years). Duration intercept and slope were both similar for frontal and central.

\section{Sex differences in spindle maturation}

For central and frontal C9 and C12 cohorts, there were sex differences in the maturation of peak-trough amplitude. For central spindle amplitude (Fig. 5) for the $\mathrm{C} 9$ and $\mathrm{C} 12$ cohorts, boys had a significantly $\left(t_{(67)}=3.02, p=0.0036\right)$ larger decline (A) and a significantly $\left(t_{(67)}=\right.$ 3.96, $p=0.0002$ ) later age of most rapid decline (M). The upper asymptote for boys was $41.39 \mu \mathrm{V}$, and amplitude decreased $12.96 \mu \mathrm{V}$ to the lower asymptote. The upper asymptote for girls was $40.24 \mu \mathrm{V}$, and amplitude decreased $8.73 \mu \mathrm{V}$ to the lower asymptote. The age of most rapid decline for boys was 14.30 years, 1.4 years later than that for girls (12.90 years). For frontal EEG data for the C9 and C12 cohorts, the upper asymptote did not differ between boys and girls (61.19 vs $\left.57.35 \mu \mathrm{V}, t_{(66)}=1.09, p=0.29\right)$. The decrease to lower asymptote trended to be larger in boys $\left(25.54\right.$ vs $20.12 \mu \mathrm{V}, t_{(66)}=1.98$, $p=0.051)$. The age of most rapid decline was again significantly later in boys than girls (14.42 vs 12.90 years, $t_{(66)}=3.71$, $p=0.0004)$.

For central channel spindle wave frequency, there was no sex effect on the intercept $\left(F_{(1,68)}=2.62, p=0.11\right)$ or the slope $\left(F_{(1,715)}=1.29, p=0.26\right)$. For frontal spindle wave frequency, the intercept did not differ by sex $\left(F_{(1,67)}=0.14, p=0.71\right)$, but the 
Table 2. Comparison of model parameters for models with and without the C6 cohort

\begin{tabular}{|c|c|c|}
\hline Spindle measure & $\begin{array}{l}\text { Central } \mathrm{C} 9 \text { and } \mathrm{C} 12 \\
\text { (estimated } 95 \% \text { Cl) }\end{array}$ & $\begin{array}{l}\text { Central C6, C9, C12 } \\
\text { (estimated 95\% Cl) }\end{array}$ \\
\hline \multicolumn{3}{|l|}{ Frequency } \\
\hline Age 6 intercept (Hz) & $11.47(11.37,11.57)$ & $11.54(11.46,11.63)$ \\
\hline Slope (Hz/yr) & $0.119(0.107,0.132)$ & $0.115(0.104,0.127)$ \\
\hline \multicolumn{3}{|l|}{ Density } \\
\hline Peak (spindles/min) & $4.90(4.81,4.99)$ & $4.87(4.77,4.97)$ \\
\hline Age of peak (yr) & $15.15(14.14,16.16)$ & $15.13(13.99,16.28)$ \\
\hline \multicolumn{3}{|l|}{ Amplitude } \\
\hline Upper asymptote $(\mu \mathrm{V})$ & $40.65(38.65,42.64)$ & $40.14(38.45,41.84)$ \\
\hline Decrease to lower $(\mu \mathrm{V})$ & $10.31(8.45,12.17)$ & $10.09(8.29,11.89)$ \\
\hline Age of fastest decline (yr) & $13.49(13.06,13.92)$ & $13.51(13.06,13.96)$ \\
\hline \multicolumn{3}{|l|}{ Duration } \\
\hline Age 6 intercept (ms) & $920.3(896.4,944.2)$ & $917.1(897.6,936.7)$ \\
\hline Slope (ms/yr) & $-6.53(-8.41,-4.64)$ & $-4.95(-6.74,-3.15)$ \\
\hline
\end{tabular}

Table 3. Comparison of frontal and central spindle characteristics

\begin{tabular}{lcc}
\hline Spindle measure & $\begin{array}{l}\text { Central C9 and C12 } \\
\text { (estimated 95\% Cl) }\end{array}$ & $\begin{array}{l}\text { Frontal C9, C12 } \\
\text { (estimated 95\% Cl) }\end{array}$ \\
\hline $\begin{array}{l}\text { Frequency } \\
\quad \text { Age 6 intercept (Hz) }\end{array}$ & $11.47(11.37,11.57)$ & $10.87(10.65,11.09)$ \\
$\quad$ Slope (Hz/yr) & $0.119(0.107,0.132)$ & $0.099(0.069,0.129)$ \\
$\begin{array}{l}\text { Density } \\
\quad \text { Peak (spindles/min) }\end{array}$ & $4.90(4.81,4.99)$ & $4.80(4.68,4.93)$ \\
$\quad$ Age of peak (yr) & $15.15(14.14,16.16)$ & $14.53(13.86,15.20)$ \\
Amplitude & & \\
$\quad$ Upper Asymptote $(\mu \mathrm{V})^{a}$ & $40.65(38.65,42.64)$ & $59.17(55.59,62.74)$ \\
$\quad$ Decrease to Lower $(\mu \mathrm{V})^{a}$ & $10.31(8.45,12.17)$ & $22.88(18.94,26.82)$ \\
$\quad$ Age of fastest decline (yr) & $13.49(13.06,13.92)$ & $13.58(13.10,14.05)$ \\
Duration & & \\
$\quad$ Age 6 intercept (ms) & $920.3(896.4,944.2)$ & $902.4(877.4,927.3)$ \\
$\quad$ Slope (ms/yr) & $-6.53(-8.41,-4.64)$ & $-7.55(-9.92,-5.18)$ \\
\hline
\end{tabular}

Mixed effects analysis produced estimates and $95 \%$ confidence limits for parameters of equations that model age-related changes. Spindle frequency intercept, spindle amplitude upper asymptote, and decrease to lower asymptote were the only measures for which the frontal and central $95 \%$ confidence intervals did not overlap (indicated by superscript a)

slope was significantly greater in girls than boys $(0.130$ vs $\left.0.065 \mathrm{~Hz} / \mathrm{yr}, F_{(1,681)}=4.94, p=0.027\right)$. There were no sex differences in the spindle density peak $\left(t_{(68)}=0.85, p=0.40\right)$ or the age of the peak $\left(t_{(68)}=0.20, p=0.84\right)$ for central or for frontal (peak $t_{(67)}=0.72, p=0.47$; age of peak $\left.t_{(67)}=1.86, p=0.067\right)$. For central spindle duration, there was no sex effect on slope $\left(F_{(1,715)}=\right.$ $0.40, p=0.53)$ or intercept $\left(F_{(1,68)}=0.02, p=0.89\right)$. For frontal spindle duration, the age 9 intercept was greater for girls than boys ( 899 vs $\left.859 \mathrm{~ms}, F_{(1,67)}=4.49, p=0.038\right)$ but the slope did not differ by $\operatorname{sex}\left(F_{(1,681)}=1.17, p=0.28\right)$

\section{Other measures}

We also fit a Gompertz model to the age-related change in Hilbert amplitude of central spindles (Figs. 2E, 3E). For central C9 and C12 cohorts, the upper asymptote was $26.47 \mu \mathrm{V}$ and decreased $6.46 \mu \mathrm{V}$ to the lower asymptote. The age of most rapid decline $(\mathrm{M})$ for central was 13.42 years. Boys had significantly larger decline $\left(8.28\right.$ vs $\left.5.45 \mu \mathrm{V}, t_{(67)}=2.93, p=0.0046\right)$ and significantly older age of most rapid decline (14.3 vs 12.9 years, $t_{(67)}=$ $3.75, p=0.0004)$.

Slow spindle frequency is the FFT bin of maximum frontal $\sigma$ power, and fast spindle frequency is the FFT bin of maximum central $\sigma$ power. Both increased linearly with age. For the C9 and C12 cohorts, slow frequency increased $0.079 \mathrm{~Hz}$ per year $\left(F_{(1,716)}=\right.$ 20.36, $p<0.0001$ ), from $11.37 \mathrm{~Hz}$ at age 9. Fast frequency (Fig. $2 F$ ) increased $0.121 \mathrm{~Hz}$ per year $\left(F_{(1,716)}=307, p<0.0001\right)$ from $12.11 \mathrm{~Hz}$ at age 9. Slow frequency age 9 intercept did not differ between boys and girls $\left(F_{(1,68)}=0.12, p=0.73\right)$, but the age-related increase in frequency was steeper in girls $(0.117$ vs $0.037 \mathrm{~Hz} / \mathrm{yr}$, $\left.F_{(1,715)}=5.70, p=0.017\right)$. Fast frequency age 9 intercept did not differ between boys and girls $\left(F_{(1,68)}=0.15, p=0.70\right)$, and the agerelated increase in frequency showed a trend $\left(F_{(1,715)}=3.67\right.$, $p=0.056)$ to be steeper in girls $(0.133$ vs $0.107 \mathrm{~Hz} / \mathrm{yr})$.

\section{Discussion}

This longitudinal study of 98 subjects (6-18 years) found strong maturational changes in sleep spindle amplitude, frequency, density, and duration. In addition, there were significant sex differences and differences in the maturational trajectories of spindles from the frontal and central derivations.

\section{Age-related change in spindle amplitude}

We previously interpreted the adolescent decline in $\sigma$ power as an indicator of synaptic pruning that would reduce the size of the neuronal pools oscillating in synchrony (Campbell and Feinberg, 2016). Other investigators have also attributed the spindle amplitude decline across adolescence to synaptic pruning (Goldstone et al., 2019). Our longitudinal design and the large number of recordings provided sufficient statistical power to investigate both nonlinear and linear models for the maturational decline in spindle amplitude. Central spindle amplitude declined steeply between ages 12 and 16 with an age of most rapid decline at the age of $\sim 13$ years. If our hypothesis is correct, it appears that synaptic pruning accelerates sharply between 12 and 16 and then slows. This pattern parallels the decline in $\delta(1-$ $4 \mathrm{~Hz}$ ) EEG power across adolescence found in this same longitudinal dataset (Feinberg and Campbell, 2013). Furthermore, the increase in spindle amplitude before age 8 years agrees with the increase in $\delta$ power before age 8 that we interpreted as synaptic proliferation continuing until this age (Feinberg and Campbell, 2013). On a basic neuroscience level, this similarity is consistent with the Steriade et al. (1993) data indicating that both $\delta$ waves and sleep spindles are generated by the same thalamocortical circuits. We note that there is, as yet, no direct support for the adolescent decline in spindle amplitude or $\delta$ power being a result of synaptic pruning. Multiple studies (Buchmann et al., 2010; Goldstone et al., 2018) have found a relation between the adolescent decline in slow wave EEG activity and the decrease in structural MRI measured cortical thickness, another measure proposed to be associate with synaptic density. However, a direct comparison of slow wave EEG activity and synaptic density in mice did not find a decrease in dendritic spines across the adolescent period when slow wave activity decreased (de Vivo et al., 2014). The authors, instead, proposed that the adolescent decrease in EEG amplitude may be associated with synaptic rearrangement and functional optimization of cortical circuits.

As mentioned previously, we decided to present a more direct amplitude measure, the peak-trough amplitude, instead of the Hilbert amplitude used by Goldstone et al. (2019). Not surprisingly, we observed similar age effects and sex effects on peaktrough amplitude and Hilbert amplitude, with similar trend fits and similar ages of most rapid decline. 

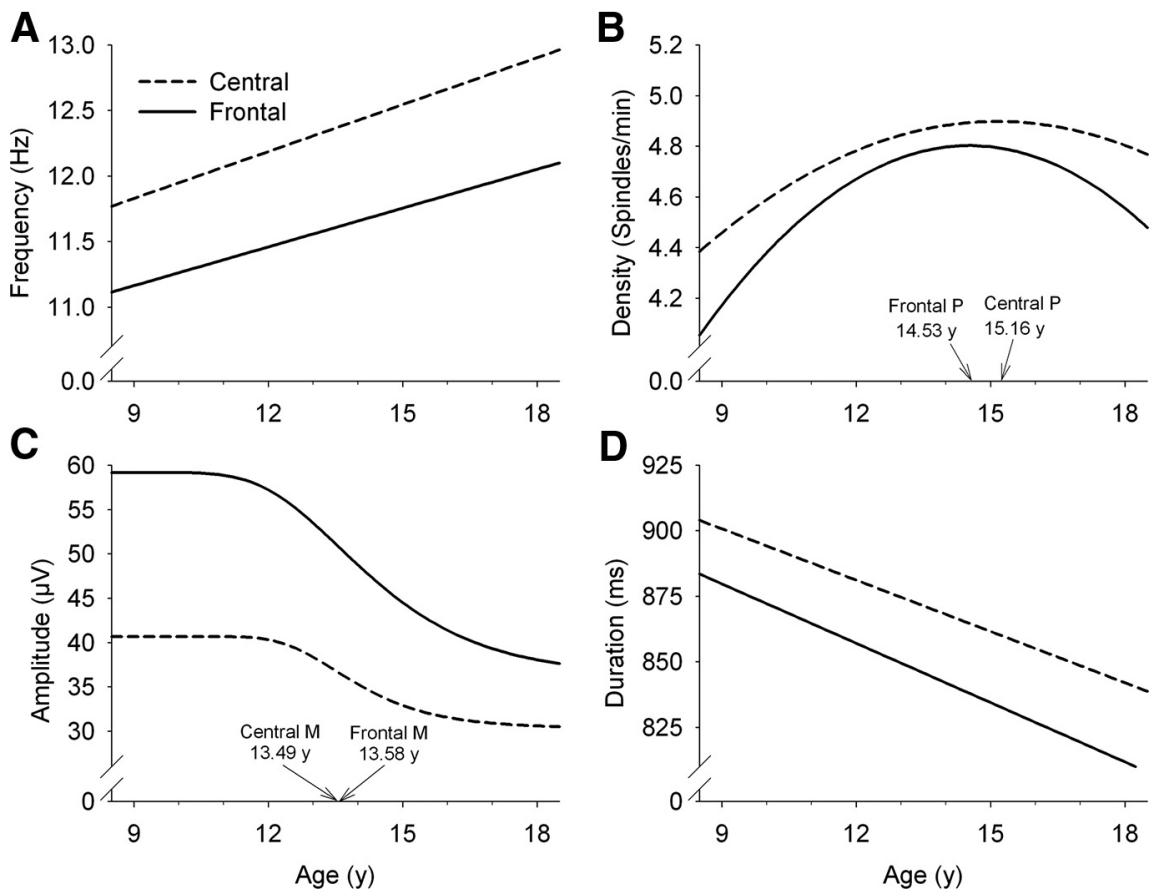

Figure 4. Comparison of age trends of central and frontal spindle measures. Trend lines are plotted for the equations that best fit the age trends for frontal (solid) and central (dotted). $\boldsymbol{A}$, Frontal spindle frequency was slower than central spindle frequency, but the age-related increases were similar. $\boldsymbol{B}$, The age of peak spindle density for frontal spindles preceded that for central spindles, but the $95 \%$ Cls (Table 3) overlapped. C, Frontal peak-trough amplitude was greater than central peak-trough amplitude, but the ages of most rapid decline did not differ significantly. $\boldsymbol{D}$, The linear decreases in central spindle duration and frontal spindle duration did not differ significantly.

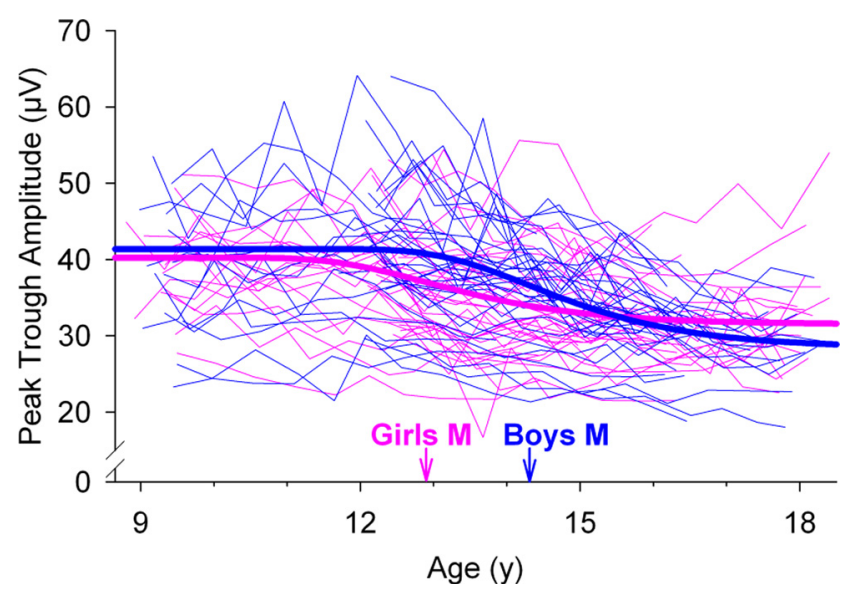

Figure 5. Sex differences in maturation of central spindle peak-trough amplitude. Each thin line indicates the maturation of spindle amplitude for an individual girl (magenta) or boy (blue). Heavy lines indicate the trend lines. The spindle amplitude decrease occurred significantly earlier in girls, with the age of most rapid decline (M) for girls preceding that for boys by $\sim 1.4$ years.

\section{Age-related change in spindle frequency}

We have previously noted that the frequency at which $\sigma$ power is at a maximum is lower for children than for adults (Campbell and Feinberg, 2016). The current data on spindle wave frequency support the hypothesis that the lower $\sigma$ frequency results from low-frequency spindle waves. Ujma et al. (2016) also report low spindle frequency for young children. The age-related change in spindle frequency was almost perfectly linear. Such linearity is remarkable in a biological measure. A linear increase in frequency has previously been reported for the age-related change in the frequency at which $\sigma$ power is at a peak (Shinomiya et al., 1999; Tarokh and Carskadon, 2010; Campbell and Feinberg, 2016; Goldstone et al., 2019). We replicate these findings and show that this linearity also holds for a direct measure of spindle wave frequency. Our interpretation of the linear age-related increase in spindle wave frequency is the same as that for our previous interpretation of the linear increase in frequency of peak $\sigma$ power (Campbell and Feinberg, 2016). Thalamocortical cell hyperpolarization determines spindle frequency, with greater hyperpolarization reducing the frequency of spindle waves (Steriade and Llinas, 1988). The degree of hyperpolarization varies with sleep depth. Spindle frequency is lowest at the start of the night and increases across the night (Andrillon et al., 2011) as sleep depth decreases. We propose that a reduction in sleep depth with age reduces hyperpolarization, thus increasing spindle wave frequency. This increase could reflect the maturation of thalamocortical circuits. As extra connections are pruned, the circuit becomes more efficient and can process data faster. It is tempting to speculate that the increase in spindle frequency signifies faster transmission. However, the linear increase in spindle frequency contrasts with the curvilinear decline in amplitude, which we interpret as a marker of the pruning process. It may, instead, be myelination that is increasing the efficiency of thalamocortical circuits and increasing spindle frequency. White matter measures have been associated with sleep spindle generation (Piantoni et al., 2013).

The linear increase in frequency cannot continue through adulthood. Purcell et al. (2017) found an increase across childhood and adolescence that eventually plateaued in adulthood. Nicolas et al.'s (2001) description of central spindle characteristics in 10 year age groups shows an increase from the teenage group through the 30 year group with a plateau or a decrease beyond age 40 years.

\section{Age-related change in spindle density}

The age effect on central spindle density is described with a parabola that peaks around age 15.1 years (95\% CI 14.1-16.2 years old). This suggests that the biological need for spindles or the capacity to generate spindles is greatest around that age. Spindles have been proposed as a protective mechanism that maintains sleep in the lighter stages by inhibiting sensory input (De Gennaro and Ferrara, 2003; Dang-Vu et al., 2010; Luthi, 2014). The sleep duration reduction and $\delta$ power decrease point to the 12 to 16 year age range as a period of rapid decline in sleep depth. The brain may adapt to this change by increasing spindle production to maintain the sleep state. Additional evidence indicates that sleep spindles may participate in circuits that generate memory and cognition (Fogel and Smith, 2011; Astori et al., 2013). Many studies have shown that high spindle density is associated with better working memory, executive function, and processing 
speed/accuracy. If correct, the need for memory processing associated with spindles is greatest in the mid-teenage years.

\section{Age-related change in spindle duration}

We replicate the decrease in spindle duration that Goldstone et al. (2019) and Purcell et al. (2017) reported. Our data do not allow us to expand on their findings because of a change in spindle duration associated with a change in the EEG recorder midway through our study. The $850-900 \mathrm{~ms}$ values that we report fit well with the $\sim 1 \mathrm{~s}$ duration for the teenage group in the Nicolas et al. (2001) study. They found a decrease in duration through the fifth decade of life. With a spindle duration $>1.5 \mathrm{~s}$ reported for 4-7 month infants (Tanguay et al., 1975), the available data point to a decrease in spindle duration across much of the human lifespan.

\section{Frontal versus central spindles}

Spindles recorded over the frontal cortex have a lower frequency than centrally recorded spindles (Jobert et al., 1992). The agerelated change in frontal spindle frequency was also linear. As expected, the line describing this increase had a lower intercept than that for central spindles. The slopes did not differ significantly; the process responsible for the maturational increase in spindle frequency appears to occur at a similar rate in the frontal and central cortices.

Frontal spindle amplitude is larger than central amplitude, which matches others' findings (Goldstone et al., 2019). Surprisingly, the age of most rapid decline was very similar (13.6 vs 13.5 years) for frontal and central spindle amplitude. Based on our $\delta$ power findings (Feinberg et al., 2011), we expected frontal spindle amplitude to show a later maturation. We, and others, have interpreted the later decline of frontal $\delta$ as later synaptic pruning in the frontal brain. Both $\delta$ power (Feinberg et al., 2011) and structural MRI data (Gogtay et al., 2004) show a back to front maturational pattern. The similar maturational age for frontal and central spindles contradicts this trend. Frontal spindles showed an earlier age of peak density, but the $95 \%$ CI overlapped that for central spindles. Despite the high statistical power of this study, we are left with the unresolved question raised by a negative finding; that is, there were no differences in frontal and central spindle maturation or we were unable to detect the differences.

\section{Sex differences in spindle maturation}

We previously found that $\delta$ power declined earlier in females, with the age of most rapid decline occurring 1.21 years earlier in girls (Campbell et al., 2012). We interpreted this as earlier synaptic pruning in females. MRI measures of cortical thickness also peak earlier in females (Giedd et al., 2015). Spindle amplitude showed a similar sex difference; the age of most rapid decline in girls preceded that of boys by $\sim 1.4$ years. Thus, the synaptic pruning that decreases spindle amplitude joins a long list of maturational events that occur earlier in females. For $\delta$ power, we found that the age of most rapid decline was strongly associated with the age of pubertal maturation measured with Tanner stage ratings (Campbell et al., 2012). It is tempting to propose that the hormonal changes of adolescence are somehow involved in synaptic pruning. However, a recent study did not find the lower $\delta$ power in girls with precocious puberty that would support that proposal (McHill et al., 2017).

Previous studies have found that spindle frequency is higher in adolescent females than males (Goldstone et al., 2019; Markovic et al., 2020). In our current study, the linear maturational trend for central spindle wave frequency did not show a sex difference in either intercept or slope. However, the slope of the age-related increase in frontal spindle wave frequency was greater in girls as was the slope for slow spindle frequency, and the slope for fast spindle frequency trended toward a steeper increase for girls. A sex difference in spindle frequency may develop across the teenage years. Spindle frequency is greater in adult females (Ujma et al., 2014).

\section{Limitations}

The spindle detection program that we used requires artifact-free frontal and central EEG. Our study was limited by having handmarked artifacts for the central channel but not for the frontal channel. Although we used FFT to detect and eliminate frontal EEG artifacts, the recordings might still have artifacts, which could be misidentified as spindles by the automatic spindle program and affect the accuracy of our frontal spindle measurements. Second, the longitudinal study duration, up to 7 years for participants in the C9 cohort, involved a recorder change midstudy. This change required us to down sample the Aura recordings to match the $200 \mathrm{~Hz}$ digitization rate of the earlier $\mathrm{H} 2 \mathrm{O}$ recordings. After down sampling, the frequency, density, and amplitude maturational trends did not show changes associated with the recorder change; however, we are not as confident about the spindle duration trend. Furthermore, the $200 \mathrm{~Hz}$ digitization rate limits the precision of the amplitude in that the actual peak of a spindle wave may occur between sample points. Similarly, the digitization rate limits precision of the location of the peak, affecting the frequency calculations. The limited number of EEG electrodes for these recordings was an attempt to balance the comfort of the participant with our desire to record as much data as possible. A larger electrode array would have allowed us to determine the maturational trend of spindle characteristics from areas of interest in addition to frontal and central. Although slow spindles have been associated with frontal EEG and fast spindles with central EEG, the $\pm 1.5 \mathrm{~Hz}$ band pass filter we used exceeds the frequency difference between fast and slow spindles. It is probable that some of the spindles recorded centrally were slow spindles from the frontal cortex and vice versa. We chose to use the $\pm 1.5 \mathrm{~Hz}$ band pass filter used in many previous studies rather than applying a narrower filter that has not been validated for spindle detection and analysis. An analysis method that better isolates fast and slow spindles may reveal developmental differences that we failed to detect. Finally, as others have suggested, the automatic spindle detection program could miscount or overcount spindles (Reynolds et al., 2018; Goldstone et al., 2019).

In conclusion, these findings further support our proposition that the brain undergoes pervasive and complex changes during adolescence. The marked decline in spindle amplitude across ages 12-16 year found here parallels previous findings for NREM $\delta$ power, emphasizing this age range as a crucial developmental period. This rise of spindle amplitude between ages 6 and 8 years also parallels our previous findings for NREM $\delta$ power and suggests that this age period also merits close scrutiny. The systematically different maturational curves for spindle amplitude, frequency, density, and duration may contribute to investigating the role of these NREM EEG oscillations in behavior and sleep mechanisms. In addition, the finding that spindle amplitude declines earlier in girls points to an interaction between brain maturation and the neuroendocrine system. Together, the findings in this longitudinal study further emphasize the value of the sleep EEG as a tool for studying brain maturation and its underlying electrophysiology. 


\section{References}

Andrillon T, Nir Y, Staba RJ, Ferrarelli F, Cirelli C, Tononi G, Fried I (2011) Sleep spindles in humans: insights from intracranial EEG and unit recordings. J Neurosci 31:17821-17834.

Astori S, Wimmer RD, Luthi A (2013) Manipulating sleep spindles: expanding views on sleep, memory, and disease. Trends Neurosci 36:738-748.

Bodizs R, Kormendi J, Rigo P, Lazar AS (2009) The individual adjustment method of sleep spindle analysis: methodological improvements and roots in the fingerprint paradigm. J Neurosci Methods 178:205-213.

Buchmann A, Ringli M, Kurth S, Schaerer M, Geiger A, Jenni OG, Huber R (2010) EEG sleep slow-wave activity as a mirror of cortical maturation. Cereb Cortex 21:607-615.

Campbell IG, Feinberg I (2009) Longitudinal trajectories of non-rapid eye movement delta and theta EEG as indicators of adolescent brain maturation. Proc Natl Acad Sci USA 106:5177-5180.

Campbell IG, Feinberg I (2016) Maturational patterns of sigma frequency power across childhood and adolescence: a longitudinal study. Sleep 39:193-201.

Campbell IG, Grimm KJ, de Bie E, Feinberg I (2012) Sex, puberty, and the timing of sleep EEG measured adolescent brain maturation. Proc Natl Acad Sci USA 109:5740-5743.

Campbell IG, Darchia N, Higgins LM, Dykan IV, Davis NM, de Bie E, Feinberg I (2011) Adolescent changes in homeostatic regulation of EEG activity in the delta and theta frequency bands during non-rapid eye movement sleep. Sleep 34:83-91.

Chugani HT, Phelps ME, Mazziotta JC (1987) Positron emission tomography study of human brain functional development. Ann Neurol 22:487-497.

Dang-Vu TT, McKinney SM, Buxton OM, Solet JM, Ellenbogen JM (2010) Spontaneous brain rhythms predict sleep stability in the face of noise. Curr Biol 20:R626-R627.

De Gennaro L, Ferrara M (2003) Sleep spindles: an overview. Sleep Med Rev 7:423-440.

Delorme A, Makeig S (2004) EEGLAB: an open source toolbox for analysis of single-trial EEG dynamics including independent component analysis. J Neurosci Methods 134:9-21.

de Vivo L, Faraguna U, Nelson AB, Pfister-Genskow M, Klapperich ME, Tononi G, Cirelli C (2014) Developmental patterns of sleep slow wave activity and synaptic density in adolescent mice. Sleep 37:689-700.

Feinberg I (1982/1983) Schizophrenia: caused by a fault in programmed synaptic elimination during adolescence? J Psychiatr Res 17:319-334.

Feinberg I, Campbell IG (2013) Longitudinal sleep EEG trajectories indicate complex patterns of adolescent brain maturation. Am J Physiol Regul Integr Comp Physiol 304:R296-R303.

Feinberg I, de Bie E, Davis NM, Campbell IG (2011) Topographic differences in the maturation of the slow wave EEG during NREM sleep. Sleep 34:325-333.

Fogel SM, Smith CT (2011) The function of the sleep spindle: a physiological index of intelligence and a mechanism for sleep-dependent memory consolidation. Neurosci Biobehav Rev 35:1154-1165.

Giedd JN, Raznahan A, Alexander-Bloch A, Schmitt E, Gogtay N, Rapoport JL (2015) Child psychiatry branch of the National Institute of Mental Health Longitudinal Structural Magnetic Resonance Imaging Study of Human Brain Development. Neuropsychopharmacology 40:43-49.

Gogtay N, Giedd JN, Lusk L, Hayashi KM, Greenstein D, Vaituzis AC, Nugent TF III, Herman DH, Clasen LS, Toga AW, Rapoport JL, Thompson PM (2004) Dynamic mapping of human cortical development during childhood through early adulthood. Proc Natl Acad Sci USA 101:8174-8179.

Goldstone A, Willoughby AR, de Zambotti M, Franzen PL, Kwon D, Pohl KM, Pfefferbaum A, Sullivan EV, Muller-Oehring EM, Prouty DE,
Hasler BP, Clark DB, Colrain IM, Baker FC (2018) The mediating role of cortical thickness and gray matter volume on sleep slow-wave activity during adolescence. Brain Struct Funct 223:669-685.

Goldstone A, Willoughby AR, de Zambotti M, Clark DB, Sullivan EV, Hasler BP, Franzen PL, Prouty DE, Colrain IM, Baker FC (2019) Sleep spindle characteristics in adolescents. Clin Neurophysiol 130:893-902.

Jobert M, Poiseau E, Jahnig P, Schulz H, Kubicki S (1992) Topographical analysis of sleep spindle activity. Neuropsychobiology 26:210-217.

Luthi A (2014) Sleep spindles: where they come from, what they do. Neuroscientist 20:243-256.

Markovic A, Kaess M, Tarokh L (2020) Gender differences in adolescent sleep neurophysiology: a high-density sleep EEG study. Sci Rep 10:15935.

McHill AW, Klerman EB, Slater B, Kangarloo T, Mankowski PW, Shaw ND (2017) The relationship between estrogen and the decline in delta power during adolescence. Sleep 40:zsx008.

Nicolas A, Petit D, Rompre S, Montplaisir J (2001) Sleep spindle characteristics in healthy subjects of different age groups. Clin Neurophysiol 112:521-527.

Piantoni G, Poil SS, Linkenkaer-Hansen K, Verweij IM, Ramautar JR, Van Someren EJ, Van Der Werf YD (2013) Individual differences in white matter diffusion affect sleep oscillations. J Neurosci 33:227-233.

Purcell SM, Manoach DS, Demanuele C, Cade BE, Mariani S, Cox R, Panagiotaropoulou G, Saxena R, Pan JQ, Smoller JW, Redline S, Stickgold R (2017) Characterizing sleep spindles in 11,630 individuals from the National Sleep Research Resource. Nat Commun 8:15930.

Purves D (1988) Body and brain: a trophic theory of neural connections. Cambridge: Harvard UP.

Rechtschaffen A, Kales A (1968) A manual of standardized terminology, techniques and scoring systems for sleep stages of human subjects. Washington, D.C: Public Health Services, U.S. Government Printing Office.

Reynolds CM, Gradisar M, Coussens S, Short MA (2018) Sleep spindles in adolescence: a comparison across sleep restriction and sleep extension. Sleep Med 50:166-174.

Shinomiya S, Nagata K, Takahashi K, Masumura T (1999) Development of sleep spindles in young children and adolescents. Clin Electroencephalogr 30: $39-43$.

Singer JD (1998) Using SAS PROC MIXED to fit multilevel models, hierarchical models, and individual growth models. J Educ Behav Stat 23:323355.

Steriade M, Llinas RR (1988) The functional states of the thalamus and the associated neuronal interplay. Physiol Rev 68:649-742.

Steriade M, McCormick DA, Sejnowski TJ (1993) Thalamocortical oscillations in the sleeping and aroused brain. Science 262:679-685.

Tanguay PE, Ornitz EM, Kaplan A, Bozzo ES (1975) Evolution of sleep spindles in childhood. Electroencephalogr Clin Neurophysiol 38:175-181.

Tarokh L, Carskadon MA (2010) Developmental changes in the human sleep EEG during early adolescence. Sleep 33:801-809.

Uchida S, Maloney T, March JD, Azari R, Feinberg I (1991) Sigma (12-15 $\mathrm{Hz})$ and delta $(0.3-3 \mathrm{~Hz})$ EEG oscillate reciprocally within NREM sleep. Brain Res Bull 27:93-96.

Ujma PP, Konrad BN, Genzel L, Bleifuss A, Simor P, Potari A, Kormendi J, Gombos F, Steiger A, Bodizs R, Dresler M (2014) Sleep spindles and intelligence: evidence for a sexual dimorphism. J Neurosci 34:1635816368 .

Ujma PP, Sandor P, Szakadat S, Gombos F, Bodizs R (2016) Sleep spindles and intelligence in early childhood-developmental and trait-dependent aspects. Dev Psychol 52:2118-2129. 